\title{
Reliability and Concurrent Validity of the Goniometer-Pro App vs a Universal Goniometer in determining Passive Flexion of Knee
}

\author{
Alberto Melian Ortiz \\ Physical therapist. FREMAP \\ Hospital (Majadahonda). PhD \\ student at Rey Juan Carlos \\ University, Mostoles (Madrid), \\ Spain. Ctra. Pozuelo, no 61 \\ 28220 Majadahonda (Madrid).
}

\author{
Sofía Laguarta Val \\ PhD in Physiotherapy. \\ Physiotherapy, Occupational \\ Therapy, Rehabilitation and \\ Phyisical Medicine Department \\ of Rey Juan Carlos University \\ (Madrid), Spain.
}

\author{
David Varillas Delgado \\ PhD Research Consultant, \\ Madrid, Spain. Ctra. Pozuelo, \\ no 6128220 Majadahonda \\ (Madrid).
}

\begin{abstract}
Purpose: Smartphones have become a staple in our society. They are essential not only for our day-to-day lives but also as a healthcare tool in the face of the emergence of a myriad of health-based applications. Analyze the reliability and concurrent validity of an iPhone app as compared with traditional goniometry.
\end{abstract}

Methods: 21 subjects of our work centre, in 2016 had 4 measurements of their degree of knee flexion for a randomly chosen knee position: 2 by universal goniometry (UG) and 2 by the Goniometer-Pro (G-Pro) application. A different evaluator made each measurement. The radiographs were used as a gold standard to measure the actual knee flexion angle.

Results: The difference between mean intra-group values was $3,148^{\circ}\left( \pm 2,669^{\circ}\right)$ and $2,476^{\circ}\left( \pm 2,638^{\circ}\right)$ for G-Pro. The difference between the mean intra-group values was $5.45^{\circ}$. Inter-observer consistency for UG was 0,990 and 0,993 for GPro; As regards validity, the values obtained were 0,976 for UG and 0,992 for G-Pro.

Conclusions: The Goniometer-Pro app seems to be a reliable and accurate tool for determining the values of knee flexion. The values obtained are slightly more accurate than those of traditional goniometry.

Implication: This study intend to promote the use of apps of systemic form in the health system, as a component integrated into the management of the health. This work not only compares the reliability of two methods of measurement, compare the reliability or validity with a gold standard, as it is the radiological measurement.

\section{Keywords}

Goniometry; smartphone app; knee flexion; knee.

\section{INTRODUCTION}

Mobile technologies, and in particular mobile applications (apps), are the ones making the most profound impact on both patients and healthcare practitioners. These mobile applications (apps) has defined as "software applications intended for use in the diagnosis or cure, mitigation, treatment or prevention of diseases that may affect the structure or any function of the body of a man or other animals [1].

A health-related APP directory has been launched in Europe in order to assist patients and practitioners in finding useful and reliable applications [2].
The new information and communication technologies have made significant inroads into medicine.

A report by the Institute for Healthcare Informatics (IMS) [3] states that over 40,000 health-based apps are available for download from Apple Store. In 2015, the number of smartphone users that had downloaded health-based apps, exceeded the 500 million mark [4].

Universal goniometry (UG) has long been regarded by both physicians and physical therapists as the most objective tool for measuring articular range of motion (ROM), thus contributing to make accurate diagnoses and monitoring the efficiency of treatment $[5,6,7,8]$. Nonetheless, some factors exist that could negatively influence the reliability and accuracy of UG such as poor identification of anatomical landmarks, malpositioning of the arms of the goniometer and inconsistencies in limb positioning $[9,10]$.

These apps may use the phone's equipped sensors (accelerometer, inclinometer, etc.) to measure physiological parameters such as limb movement [1]. This technology is gaining increasing ground in clinical practice, as it constitutes a fast, accurate and easy-to-interpret tool [11,12,13,14,15]. Moreover, these applications can be used by patients themselves outside the clinical environment [16].

The purpose of this study is to analyze the reliability and concurrent validity of a health-related iPhone app as compared with traditional UG for measuring a given degree of knee flexion.

\section{MATERIAL AND METHODS}

A non-experimental double blind comparative and descriptive study was carried out by the Radiology and Physiotherapy units of the FREMAP Hospital in Majadahonda (Madrid, Spain) in 2016. The study was granted approval by the Hospital's scientific and ethical committee.

The study was performed by five physical therapists with over 15 years' experience in the field of musculoskeletal treatment plus a researcher responsible for statistical analysis. Four of the physical therapists were in charge of the measurements while the fifth was responsible for measuring the subject's degree of knee flexion as measured by $\mathrm{x}$-ray. Two of the evaluators used the Goniometer-Pro (G-Pro) health-based iPhone app while the other two used UG. The first two had never used the G-Pro app, whereas the last two were in the habit of using UG. The method used to measure the degree of knee flexion with UG was as follows: the fulcrum of the 
goniometer was placed on the lateral condyle of the left femur, the fixed arm of the goniometer was situated on the lateral aspect of the femur pointing to the greater trochanter, and the movable arm was placed on the lateral aspect of the leg pointing to the lateral malleolus [17]. As regards the GPro app, it bases its measurements in tibial inclination with respect to horizontal, being that of the orientation of femur.

The study sample comprised 21 subjects who were asked to sign an informed consent form. Subjects were placed in the prone position on an examination table in the radiology room. Next, a physical therapist placed the left knee at a randomly chosen degree of flexion. This flexion was stood still through a few cots and pillows to prevent any alteration in the positioning of the knee.

Subsequently, 4 measurements of the subjects' passive of knee flexion were carried out: two measurements were made by UG and two with the G-Pro app, shown on Figure 1.

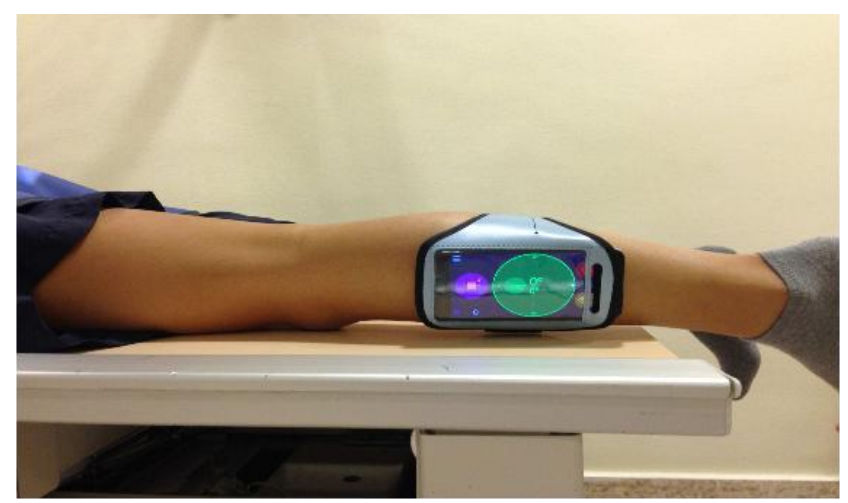

Figure 1. Measurement using the G-Pro app.

Each evaluator noted the value obtained on a specificallydesigned template without disclosing any information to the others. Finally, Figure 2 shows all subjects were subjected to a lateral radiograph, with the knee joint in the initial position.

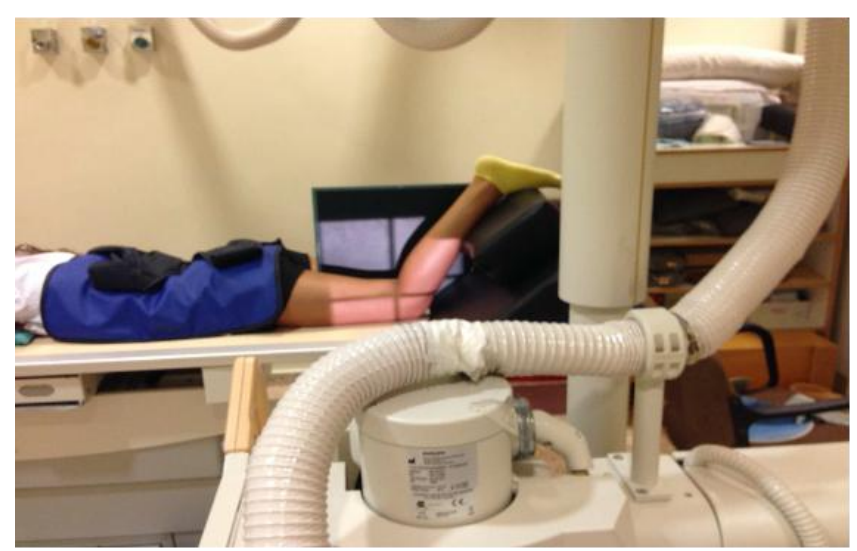

Figure 2. Lateral x-ray of the left knee.

Such radiographs were used by the fifth physical therapist to measure the real value of passive knee flexion on the basis of the orientation of the anatomical axis of the femur and the tibia is given in Figure 3.

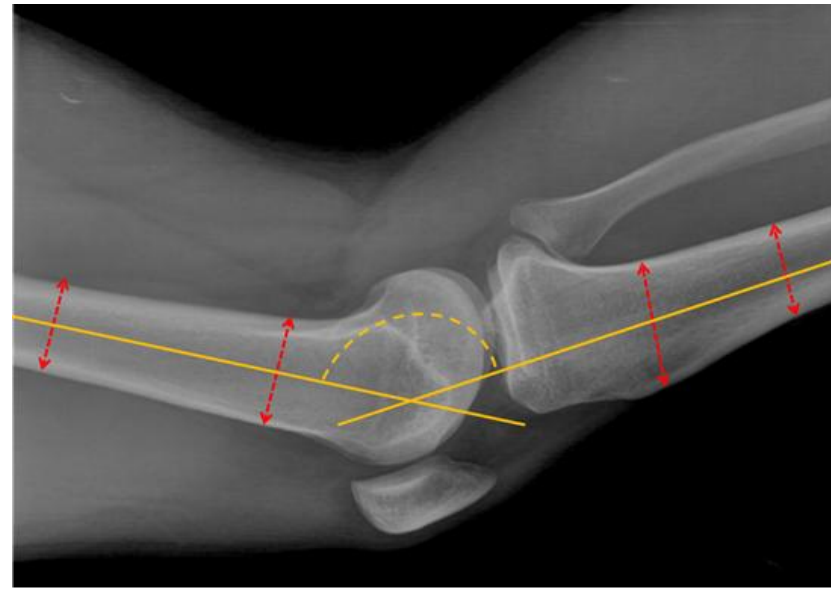

Figure 3. X-ray-based measurements.

Once all four goniometric measurements were performed and the corresponding lateral $\mathrm{x}$-ray was analyzed, the values obtained by the five physical therapists were e-mailed to the sixth researcher, who was in charge of carrying out the statistical analysis without knowledge of what measurement group the each value belonged to.

Analysis of the data was performed using SPSS $20.0 \AA$ software for Windows. This analysis made it possible to obtain values for inter-observer consistency, using Cronbach's alpha. The intra-class correlation coefficient (ICC) was used for determining the accuracy of the two measurement tools as compared with x-ray, while the Pearson correlation coefficient was calculated to establish the correlation between the X-ray measurements and the average values obtained by the two measurement tools.

This research did not receive any specific grant from funding agencies in the public, commercial, or not-for-profit sectors.

\section{RESULTS}

Descriptive data is shown on Table 1 . The difference between the mean intra-group values was $3,148^{\circ}\left( \pm 2,669^{\circ}\right)$ and $2,476^{\circ}$ $\left( \pm 2,638^{\circ}\right)$ for G-Pro. The difference between the mean intragroup values was $5,45^{\circ}$.

Table 1: Data of measurements with Universal Goniometer (UG) and Goniometer-Pro (G-Pro) in passive flexion of knee.

\begin{tabular}{|c|c|c|c|c|c|c|c|}
\hline $\begin{array}{l}\text { Sub } \\
\text { ject }\end{array}$ & $\begin{array}{l}U G- \\
1\left(\left(^{\circ}\right)\right.\end{array}$ & $\begin{array}{l}U G- \\
2\left(\left(^{\circ}\right)\right.\end{array}$ & $\begin{array}{c}U G- \\
1 / U G-2 \\
\left({ }^{o}\right)\end{array}$ & $\begin{array}{c}G- \\
\text { Pro-1 } \\
\left({ }^{o}\right) \\
\end{array}$ & $\begin{array}{c}G- \\
\text { Pro-2 } \\
\left({ }^{o}\right) \\
\end{array}$ & $\begin{array}{c}\text { G-Pro- } \\
1 / G-P r o-2 \\
\left({ }^{o}\right) \\
\end{array}$ & $\begin{array}{l}\boldsymbol{R x} \\
\left(^{o}\right)\end{array}$ \\
\hline 1 & 27 & 25 & 26 & 20 & 18 & 19 & 22 \\
\hline 2 & 46 & 41 & 43,5 & 37 & 40 & 38,5 & 36 \\
\hline 3 & 44 & 46 & 45 & 36 & 37 & 36,5 & 34 \\
\hline 4 & 86 & 84 & 85 & 76 & 75 & 75,5 & 80 \\
\hline 5 & 64 & 62 & 63 & 60 & 61 & 60,5 & 60 \\
\hline 6 & 54 & 60 & 57 & 52 & 56 & 54 & 46 \\
\hline 7 & 95 & 88 & 91,5 & 85 & 84 & 84,5 & 90 \\
\hline 8 & 36 & 29 & 32,5 & 25 & 25 & 25 & 22 \\
\hline 9 & 62 & 61 & 61,5 & 57 & 54 & 55,5 & 50 \\
\hline 10 & 71 & 69 & 70 & 67 & 65 & 66 & 65 \\
\hline 11 & 88 & 89 & 88,5 & 99 & 86 & 92,5 & 90 \\
\hline
\end{tabular}




\begin{tabular}{|c|c|c|c|c|c|c|c|}
\hline $\mathbf{1 2}$ & 18 & 21 & 19,5 & 17 & 20 & 18,5 & 14 \\
\hline $\mathbf{1 3}$ & 71 & 71 & 71 & 65 & 66 & 65,5 & 64 \\
\hline $\mathbf{1 4}$ & 53 & 46 & 49,5 & 44 & 43 & 43,5 & 48 \\
\hline $\mathbf{1 5}$ & 19 & 18 & 18,5 & 18 & 16 & 17 & 14 \\
\hline $\mathbf{1 6}$ & 55 & 56 & 55,5 & 52 & 48 & 50 & 50 \\
\hline $\mathbf{1 7}$ & 70 & 69 & 69,5 & 63 & 61 & 62 & 70 \\
\hline $\mathbf{1 8}$ & 39 & 38 & 38,5 & 30 & 28 & 29 & 30 \\
\hline $\mathbf{1 9}$ & 56 & 65 & 60,5 & 52 & 50 & 51 & 54 \\
\hline $\mathbf{2 0}$ & 47 & 52 & 49,5 & 48 & 45 & 46,5 & 45 \\
\hline $\mathbf{2 1}$ & 61 & 59 & 60 & 51 & 50 & 50,5 & 52 \\
\hline
\end{tabular}

UG-1: Values from observer 1 of Universal Goniometer (degrees).

UG-2: Values from observer 1 of Universal Goniometer (degrees).

UG-1/UG-2: Median values from observer 1 and 2 of Universal Goniometer (degrees)

G-Pro-1: Values from observer 1 of app G-Pro (degrees).

G-Pro-2: Values from observer 2 of app G-Pro (degrees).

G-Pro-1/G-Pro-2: Median values from observer 1 and 2 of app G-Pro (degrees).
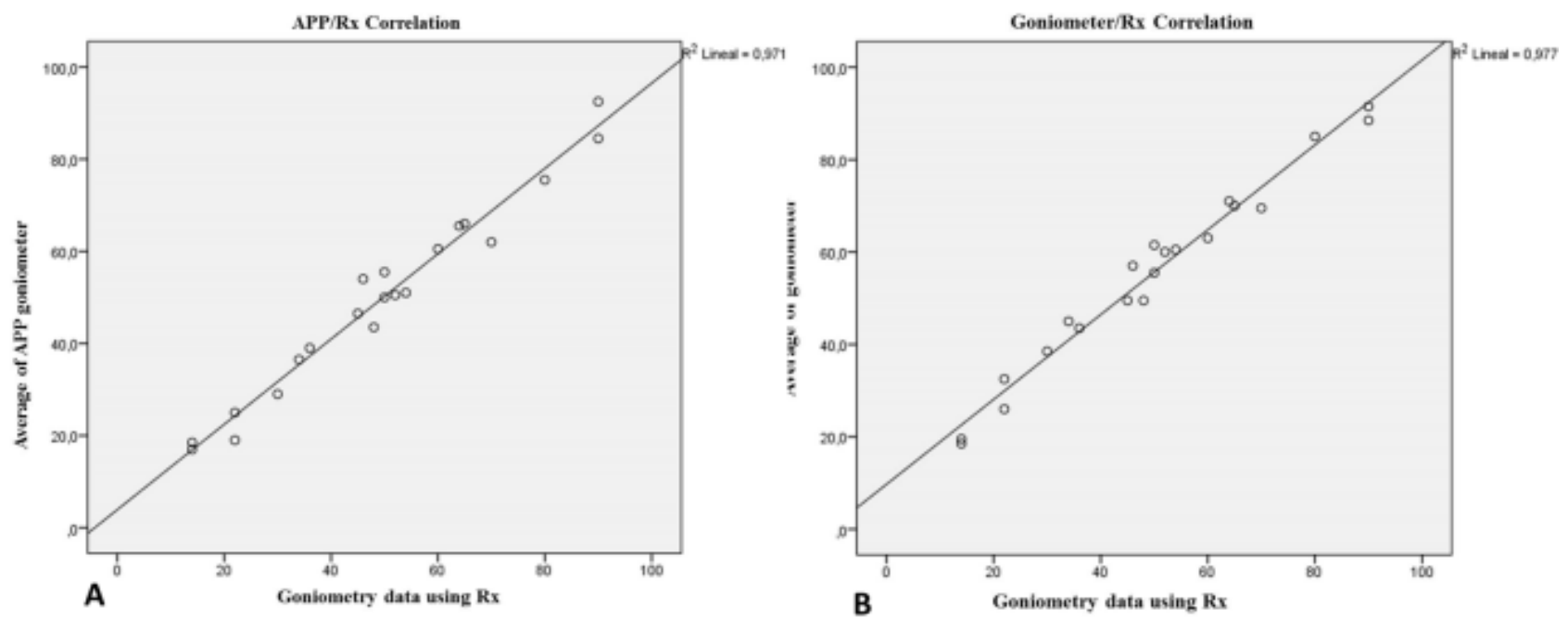

Figure 4. A. G-Pro/x-ray correlation and B. UG/x-ray correlation.

\section{DISCUSSION}

Mobile health is quickly becoming a mainstay of healthcare around the world, including developing countries [18]. As a technology that is available to "everyone, anywhere, anytime", it provides huge opportunities for innovation in healthcare. In this respect, applications conceived for measuring articular ROM are a straightforward and convenient tool that offers benefits to both patients and practitioners. As shown by this study, although both UG and G-Pro are highly reliable methods $(0,990$ and 0,993 respectively), Cronbach's alpha demonstrates a slight advantage in favor of the app. In terms of accuracy, both techniques are highly accurate but the mobile app G-Pro exhibited a slightly higher value $(0,976$ as compared with $0,992)$, with a quasi-perfect correlation $(0,985)$.
Rx: Values from radiographs (degrees).

\subsection{Reliability}

For UG, Cronbach's alpha was 0,990 and ICC was 0,990 . For G-Pro, Cronbach's alpha was 0,993 and ICC was 0,993 .

\subsection{Validity}

The accuracy of the different measurements was analyzed comparing the mean values obtained using each of the measurement techniques with the values obtained from the radiographs. The purpose was to determine which of the two techniques provided values that came closer to the real values as calculated from the radiographs. On this basis, the accuracy of the techniques was established at 0,976 for UG and 0,992 for G-Pro.

In addition, a graphic representation of the values measured by the two techniques under study (UG and G-Pro) and the xray values was made in order to determine whether there was a strong correlation between them. For UG, the correlation coefficient between the mean values from both evaluators and the $\mathrm{x}$-ray measurements was 0,977 ; the Pearson correlation coefficient was 0,988 , in Figure 4A. For G-Pro, the correlation coefficient between the mean values from both evaluators and the $\mathrm{x}$-ray measurements was 0,971; the Pearson correlation coefficient was 0,985 , in Figure 4B. 
significant differences between the two measurement tools (Pearson correlation coefficient 0,96 vs. 0,98 ), confirm that both systems are equally valid. Like Milanese [19] and Jones [20] not compare the values obtained by the two measuring tools with the actual knee mobility as recorded by $\mathrm{x}$-ray.

On the basis of a cohort of five subjects, Ockendon and Gilbert [21] compare the validity of a purpose-built mobile application to that of a traditional goniometer for measuring knee range of motion. This results show that the values obtained from both measurement instruments are closely correlated, albeit with slight differences in favor of the mobile app (inter-observer correlation: was 0,994 vs. 0,952 and intraobserver correlation: was 0,982 vs. 0,927); the mean difference between the two is only $-0,4^{\circ}$. In this study, the difference was $5,45^{\circ}$. This value is not available in the work of Ockendon and Gilbert as the authors not compare the results obtained from the measuring instruments with the real flexion value as measured by $\mathrm{x}$-ray [21]

Other studies, such as the one by Ferretti [22], validate the clinical use of a smartphone application (SmartJoint) for measuring anterior tibial translation during Lachman's test in 35 knees with a deficient anterior cruciate ligament. In this study, two evaluators compared the values obtained from the app with those obtained from applying the KT-1000 arthrometer to both knees, one of them with a deficient ACL. The authors conclude that the performance of the new application is comparable and highly correlated with the measurements from the arthrometer (mean ICC for the knee with a deficient ACL was 0,987).

But the positive results obtained by mobile applications are in no way restricted only to the measurement of knee mobility. Several authors have found comparable results using similar apps for other joints such as wrist [23], fingers [8,9], shoulder [24,25], lumbar spine [26], or even to analyze range of motion in patients who have suffered a stroke [27].

The data presented so far provides ample evidence that mHealth and, specifically, health-based apps are likely to result in an improvement in quality of care and greater efficiency in the evaluations and examinations made by health care practitioners. However, further studies are required to determine the extent to which the new technologies conceived to measure range of motion may lead to cost savings in healthcare.

According to an analysis of the Kinsey Global Institute published in 2011 [28], health-based apps could save the European public health sector 250 billion euros and the United States' public health sector around 300 billion dollars. Moreover, as a result of the lower cost of mobile technologies and their widespread embrace, the use of apps is on the rise in places where traditional technology is virtually absent. In desolate and isolated regions of the world, mobile devices facilitate remote patient care, telemedicine and emergency intervention. A report by PricewaterhouseCoopers [29], published in 2012, claims that mobile applications could save over a million lives in Sub-Saharan Africa in the next five years and save the European Union up to 100 billion euros until 2017. In Spain, a recent study on the information society, published by the Telefonica Foundation [30], states that the use of mobile technologies may reduce the per capita healthcare bill in Europe by $18 \%$ in 2017. This reduction could be of up to $35 \%$ in the case of chronic patients.

Therefore mHealth can be considered a key factor in the progression to a more sustainable healthcare, resulting in improvements in efficacy and efficiency, reducing costs and meeting the most basic needs of our society at a time when aging and chronic diseases have emerged as a formidable challenge.

The study was several limitations; no symptomatic patients to compare the measurements with respect to the asymptomatic patients in the study, sample size was not high enough to draw conclusions from the outcomes.

\section{CONCLUSIONS}

In conclusion both the Goniometer-Pro app and the universal goniometer are reliable tools for accurately measuring the range of motion of a knee. The results presented of the mobile application were slightly more accurate than those of traditional goniometry, which seems to indicate that the G-Pro app is a useful tool to measure the arc of movement of the knee. In addition, the convenience and portability of a mobile application greatly simplifies ROM determination. mHealth is a groundbreaking technology that is bound to radically change the way medicine is practiced. But this should come as no surprise since it has long been known that almost $100 \%$ of medical knowledge is renewed every 20 years [18]. A day will probably come when physicians, pharmacists, physical therapists and other healthcare practitioners will prescribe, apart from the most appropriate treatments for each case, a series of relevant, reliable and reputed online resources [18].

Future scope of this work will be the verification by other studies of the performance of Goniometer-Pro app and the universal goniometer in patients with knee injury and its comparison with symptomatic patients, checking whether the validity data shown in this study can be extrapolated into rehabilitation services.

\section{ACKNOWLEDGMENTS}

Our thanks to the radiodiagnostic service of the Hospital and to Cleofas Rodríguez and Luis Palomeque for their invaluable support.

\section{REFERENCES}

[1] United States government agency responsible for regulating food, drugs, cosmetics, medical devices, biologic products and blood derivatives (FDA), United States of America, 2016

[2] European Directory of Health apps 2012-13. A review by patients groups and empowered consumers, 2015.

[3] Institute for Healthcare Informatics (IMS), 2015.

[4] Research 2 Guidance. Mobile Health Market Report, 2013-2017, 2016

[5] Gajdosik, R., Bohannon, R. Clinical measurement of range of motion: Review of goniomtery emphasizing reliabilty and validity. Physical Therapy. 1987;67(12):1867-72.

[6] Lea, R.D., Gerhardt, J.J. Current concepts review: Range of motion measurements. J Bone Joint Surg Am 1995;77(1):784-98

[7] Croxford, P., Jones, K., Barker, K. Inter-tester comparison between visual estimation and goniometric measurement of ankle dorsiflexion. Physiother Theory Pract. 2009;14(6):107-13.

[8] Bruton, A., Ellis, B., Goddard, J. Comparison of visual estimation and goniometry for assessment of 
metacarpophalangeal joint angle. Physiother. 1999;85:201-8

[9] Nawoczenski, D.A., Baumhauer, J.F., Umberger, B.R. Relationship between clinical measurements and motion of the first metatarsophalangeal joint during gait. J Bone Joint Surg Am. 1999;81(3):370-6.

[10] Biedert, R.M., Warnke, K. Correlation between the Q angle and the patella position: a clinical and axial computed tomography evaluation. Arch Orthop Trauma Surg. 2001; 121(6):346-9.

[11] Kailas, A., Chong, C.C., WatanabeM F. From mobile phones to personal wellness dashboards. IEEE Pulse. 2010;1(1):57-63.

[12] Terry, M. Medical applications for smartphones. Telemed J and e-health. 2010;16:17-22.

[13] Free, C., Phillips, G., Galli, L., Watson, L., Felix, L., Edwards, P., Patel, V., Haines, A. The effectiveness of mobile-health technology-based health behaviour change or disease management interventions for health care consumers: a systematic review. PLoS Med. 2013;10(1):e1001362.

[14] Koehler, N., Vujovic, O., McMenamin, C. Are individuals more accepting of the internet than mobile phone applications being used in clinical practice?. J Mob Technol Med. 2013;2(1):14-21.

[15] Milani, P., Coccetta, C.A., Rabini, A., Sciarra, T., Massazza, G., Ferriero, G. Mobile Smartphone applications for body position measurement in rehabilitation: a review of goniometric tools. PM\&R. 2014;6(11):1038-43.

[16] Kim, T.S., Park, D.D., Lee, Y.B., Han, D.G., Shim, J.S., Lee, Y.J., Kim, P.C. A study on the measurement of wrist motion range using the iPhone 4 gyroscope application. Ann Plast Surg 2014;73(2):215-8.

[17] Norkin, C.C., White, D.J. 2005. Goniometria: evaluación de la movilidad articular. Madrid: Marban Libros.

[18] The app Intelligence. Report on the 50 top Spanishlanguage health-based apps, 2014.

[19] Milanese, S., Gordon, S., Buettner, P., Flavell, C., Ruston, S., Coe, D., O'Sullivan, W., McCormack, S. Reliability and concurrent validity of knee angle measurement: smartphone app versus universal goniometer used by experienced and novice clinicians. Man Ther. 2014;19(6):569-74.

[20] Jones, A., Sealey, R., Crowe, M., Gordon, S. Concurrent validity and reliability of the simple goniometer iPhone app compared with Universal Goniometer. Physiother Theory Pract. 2014;30(7):512-6.

[21] Ockendon, M., Gilbert, R.E. Validation of a novel smartphone accelerometer-based knee goniometer. J Knee Surg. 2012;25(4):341-5.

[22] Ferretti, A., Valeo, L., Mazza, D., Muliere, L., Iorio, P., Giovannetti, G., Conteduca, F., Iorio, R. Smartphone versus knee ligament arthrometer when size does not matter. Int Orthop. 2014;38(10):2197-9.

[23] Kuegler, P., Wurzer, P., Tuca, A., Sendlhofer, G., Lumenta, D.B., Giretzlehner, M., Kamolz, L.P. Goniometer-apps in hand surgery and their applicability in daily clinical practice. Safety in Health, 2015;1:11.

[24] Werner, B.C., Holzgrefe, R.E., Griffin, J.W., Lyons, M.L., Cosgrove, C.T., Hart, J.M., Brockmeier, S.F. Validation of an innovative method of shoulder range-ofmotion measurement using a smartphone clinometer application. J Shoulder Elbow Surg. 2014 Nov;23(11):e275-82

[25] Johnson, L.B., Sumner, S., Duong, T., Yan, P., Bajcsy, R., Abresch, R.T., de Bie, E., Han, J.J. Validity and reliability of smartphone magnetometer-based goniometer evaluation of shoulder abduction: A pilot study. Man Ther. 2015;20(6):777-82.

[26] Yoon, J.O., Kang, M.H., Kim, J.S., Oh, J.S. The effects of gait use of smartphone on repositioning error and curvature of the lumbar spine. J Phys Ther Sci. 2015;27(8):2507-8

[27] Lee, W.W., Yen, S.C., Tay, A., Zhao, Z., Xu, T.M., Ling, K.K., Ng, Y.S., Chew, E., Cheong, A.L., Huat, G.K.. A smartphone-centric system for the range of motion assessment in stroke patients IEEE J Biomed Health Inform. 2014;18(6):1839-47.

[28] Kinsey Global Institute, 2011.

[29] Touching lives through mobile health. Assessment of the global market opportunity, 2012.

[30] Report on the Society of Information in Spain by Fundación Telefónica, 2016. 